\title{
Método de dimensionamento espacial aplicado para vazamentos hipotéticos de depósitos de rejeitos de mineração
}

Spatial sizing method applied for hypothetical leaks of mining taillings deposits

Método de dimensión espacial aplicado para fugas hipotéticas de depósitos de relaves mineras

Recebido: 27/06/2021 | Revisado: 07/07/2021 | Aceito: 12/07/2021 | Publicado: 22/07/2021

Lariana Têka Barra de Medeiros

ORCID: https://orcid.org/0000-0002-9768-5100 Universidade Federal do Pará, Brasil

E-mail: lariana.md@gmail.com

Aline Maria Meiguins de Lima

ORCID: https://orcid.org/0000-0002-0594-0187 Universidade Federal do Pará, Brasil E-mail: ameiguins@ufpa.br

\begin{abstract}
Resumo
O setor mineral apresenta grande importância na economia brasileira, entretanto pode ser visto como um vilão na área ambiental, sobretudo devido aos impactos negativos ocasionados pela disposição dos rejeitos oriundos das atividades minerais. Com o intuito de auxiliar no planejamento de ações de gestão riscos, este estudo testou um modelo simplificado adaptado para geração de cenários de vazamento hipotético de um depósito de rejeitos de alumina, localizado no município de Barcarena-PA. As simulações foram realizadas com base no método LNEC com adaptações para análise do depósito de rejeitos, para isso foram feitos testes para definir os intervalos de descargas capazes de gerar manchas com diferentes graus de risco à inundação (alto, médio e baixo) ao longo do Igarapé Murucupi. Os resultados indicaram o risco à inundação das localidades de Vila dos Cabanos, Itupanema e Laranjal. Conclui-se que o método LNEC com ajustes pode ser utilizado na geração da mancha de inundação de depósitos de rejeito para identificação de zonas de risco e indicação de áreas prioritárias para ações do poder público, sendo, portanto, um importante instrumento de gestão de risco.
\end{abstract}

Palavras-chave: Depósitos de rejeito; Mineração; Risco à inundação.

\begin{abstract}
The mineral sector have great importance in the Brazilian economy, however it can be seen as a villain in the environmental area, mainly due to the negative impacts caused by the disposal of tailings from mineral activities. In order to assist in the planning of risk management actions, this study tested a simplified model adapted to generate hypothetical leakage scenarios from an alumina tailings deposit, located in the city of Barcarena-PA. The simulations were carried out based on the LNEC method with adaptations for analyzing the tailings deposit. For this, tests were carried out to define the discharge intervals capable of generating spots with different degrees of risk to flooding (high, medium and low) throughout the Murucupi River. The results indicated the risk of flooding in Vila dos Cabanos, Itupanema and Laranjal. It is concluded that the LNEC method with adjustments can be used in the generation of the flooding spot of tailings deposits to identify risk areas and indicate priority areas for public sector actions, being, therefore, an important risk management tool.
\end{abstract}

Keywords: Tailings deposits; Mining; Flood risk.

\section{Resumen}

El sector minero es de gran importancia en la economía brasileña, mientras puede ser visto como un villano en el área ambiental, principalmente por los impactos negativos provocados por la disposición de relaves de actividades mineras. Con el fin de ayudar en la planificación de las acciones de gestión de riesgos, este estudio probó un modelo simplificado adaptado para generar escenarios hipotéticos de fuga de un depósito de relaves de alúmina, situado en la ciudad de Barcarena-PA. Las simulaciones se realizaron con base en el método LNEC con adaptaciones para el análisis del depósito de relaves, para eso se realizaron pruebas para definir los intervalos de descarga capaces de generar puntos con diferentes grados de riesgo de inundación (alto, medio y bajo) a lo largo del Río Murucupi. Los resultados indicaron el riesgo de inundaciones en Vila dos Cabanos, Itupanema y Laranjal. Se concluye que el método LNEC con ajustes puede ser utilizado en la generación del mapa de inundación de depósitos de relaves para identificar zonas de riesgo e indicar áreas prioritarias para acciones del sector público, siendo, por tanto, una importante herramienta de gestión de riesgos.

Palabras clave: Depósitos de relaves; Minería; Riesgo de inundación. 


\section{Introdução}

A importância econômica da indústria mineral no Brasil é perceptível desde os tempos coloniais, quando as incursões dos bandeirantes em busca de metais preciosos definiram as rotas para a ocupação do interior do país. Com o aumento da ocupação do território e do conhecimento geológico, novos depósitos minerais foram descobertos, representando impacto importante na economia nacional e estimulando o processo de industrialização brasileiro. $\mathrm{O}$ minério que mais tem se destacado na produção nacional é o ferro, cuja produção se concentra, sobretudo, nos estados do Pará e de Minas Gerais (ANM, 2020).

O Pará apresenta um grande potencial para se tornar um dos maiores centros mineradores mundiais. No ano de 2018, 88\% do total de exportações do Pará se referiram as indústrias de mineração e de transformação mineral, totalizando US\$ 13,725 bilhões. Dessa forma o setor mineral se destaca como sendo o grande vetor do comércio exterior paraense, tendo o ferro o principal produto de exportação (Simineral, 2019).

Se por um lado, deve-se considerar a relevância socioeconômica da atividade mineral, por se tratar de um setor gerador de matéria-prima para produção de bens e serviços cada vez mais demandados, além de empregos, diretos e indiretos, renda e tributos. Por outro lado, a indústria mineral gera mudanças no uso do solo, muitas vezes provocando reassentamentos ou impondo necessidades de readaptação das comunidades, que são obrigadas a conviver com a mineração em sua vizinhança, alterando seu modo de vida (Ribeiro \& Silva, 2018).

Outro impacto negativo ocasionado por muitas indústrias minerais é a produção de elevada quantidade de rejeitos, os quais podem ser dispostos em diferentes tipos de estruturas, tais como, cavas exauridas, minas subterrâneas, pilhas, depósitos, ou barragens de rejeitos. Independente da estrutura utilizada para contenção, a disposição de rejeitos sempre pode apresentar riscos.

Em termos estruturais existem diferenças entre uma barragem e um depósito de rejeitos. A barragem visa a contenção de água e sólidos livres e em suspensão que são oriundos do beneficiamento do minério (compondo estruturas de portes e tamanhos variados dependentes das condições topográficas de instalação); já os depósitos de rejeitos podem ter diversas origens e formatos (podendo ser delimitados por diques de contenção ou dispostos em áreas livres e até mesmo de preenchimento subterrâneo), dependendo do tratamento que é dado a este, podendo ser: filtrado com posterior empilhamento, com espessamento para sua disposição final em pasta e por co-disposição com o estéril da mina, onde podem ser inclusive utilizadas cavas exauridas ou áreas mineradas encerradas (Stela et al., 2020).

O município paraense de Barcarena abriga grandes indústrias do setor minero-metalúrgico e se configura também como uma zona portuária. Associada a tais atividades, desde o ano 2000, ocorreram 26 acidentes ambientais na cidade, sendo três relacionados a vazamentos de rejeitos de beneficiamento de alumina provenientes da empresa Hydro Alunorte (Pará, 2018). Além do fator recorrência de acidentes, o que também é preocupante no local é a existência de comunidades próximas às áreas de produção industrial, demandando ainda mais cuidado com relação a gestão de riscos das empresas situadas na região.

Uma técnica que permite avaliar o risco de um possível vazamento ou rompimento de uma estrutura que armazena rejeitos é o mapa de inundação. Segundo a Lei n 14.066/2020, o mapa de inundação é o produto do estudo de inundação que consiste na delimitação geográfica georreferenciada das áreas potencialmente afetadas por eventual ruptura ou vazamento de barragem e seus possíveis cenários associados. Essa ferramenta tem a finalidade de permitir a notificação eficiente e o planejamento da evacuação de áreas afetadas em caso de acidente.

Para gerar os mapas de inundação podem ser utilizados métodos tradicionais, como o HEC-RAS, ou modelos simplificados (Kumar et al., 2017; Santos et al., 2020; Tschiedel et al., 2020). O HEC-RAS é um dos modelos mais utilizados para ensaios de ruptura de barragens e é baseado na equação unidimensional da conservação da energia (Pereira et al., 2017). O processamento utilizando esse software é composto por uma série de etapas que demandam tempo e dedicação do analista. 
Os métodos simplificados, por sua vez, são mais simples, mais rapidamente processados, demandam poucos danos de entrada, no entanto podem resultar em manchas não tão realistas. Um modelo desse tipo foi elaborado pelo Laboratório Nacional de Engenharia Civil (LNEC) de Portugal. Esse método executa processamentos automatizados para geração da mancha de inundação associada a rompimento de estruturas como barramentos, utilizando os dados altimétricos do SRTM (Shuttle Radar Topography Mission) com resolução espacial de 30m e como dados de entrada: a altura da barragem, a cota de coroamento e o volume do reservatório.

Nesse sentido, este estudo testou uma adaptação do modelo simplificado LNEC para geração de cenários de vazamento hipotético de um depósito de rejeitos de alumina, localizado no município de Barcarena, no estado do Pará, com o intuito de auxiliar no planejamento de ações de gestão riscos.

\section{Procedimentos Aplicados ao Dimensionamento Espacial de Vazamentos Hipotéticos de Barragens}

As manchas de inundação, geradas simulando o rompimento ou o vazamento de estruturas que comportam os rejeitos, auxiliam na delimitação das áreas de risco. Nesta proposta, é aplicado um método simplificado com o intuito de auxiliar profissionais impossibilitados de usarem procedimentos mais detalhados em virtude, principalmente, das limitações de dados, de ferramentas computacionais ou devido à necessidade de estimativa rápida para áreas inundáveis. Esses métodos estimam a propagação do hidrograma de ruptura a jusante, sem o uso de modelos hidráulicos e hidrodinâmicos, e são baseados sobretudo nas equações de balanço de massa (Collischonn \& Tucci, 1997; Sousa et al., 2021).

Apesar dessas metodologias não garantirem um resultado com acurácia, elas auxiliam na gestão de risco, uma vez que permitem agilizar a identificação de áreas de risco à inundação decorrentes do rompimento ou do vazamento de estruturas de barramento. Uma técnica simplificada que tem sido utilizada no Brasil e que é sugerida pela Agência Nacional de Águas (ANA) para classificação de barragens é o método desenvolvido por pesquisadores do LNEC. As formulações técnicas que fundamentam esse método são apresentadas a seguir, tendo como base a publicação da ANA (2017).

A primeira etapa consiste em estabelecer a extensão do rio a ser analisada, ou seja, a distância máxima (Dmax), calculada com base no volume máximo do reservatório (Vmax), conforme a equação (1), se Vmax $\leq 1000$ hm³ (Figura 1). Para reservatórios com volume máximo maior que $1.000 \mathrm{hm}^{3}$, utiliza-se a distância máxima de $100 \mathrm{Km}$. Em seguida é feita a estimativa da vazão máxima associada à ruptura da barragem, usando as fórmulas de Froehlich (equação 2) e MMC (equação 3). A concepção teórica parte do princípio que com a propagação da onda de rompimento, ocorre um amortecimento do hidrograma, caracterizado pela redução da vazão de pico e alargamento da onda (Rossi et al., 2021).

Após o estabelecimento da vazão máxima na seção da barragem, o trecho do rio em análise é dividido em 21 seções para que seja estimada a vazão de pico de cada seção transversal analisada, estabelecendo o amortecimento da vazão de pico ao longo do vale. Para calcular as vazões de pico em cada uma das seções transversais ao longo do vale, são utilizadas a equação 4 para volumes de reservatório superiores a $6,2 \mathrm{hm}^{3} \mathrm{e}$ a equação 5 para reservatórios inferiores (Figura 1).

Considerando as características físicas da barragem (tais como, altura, cota de coroamento e volume do reservatório) e o perfil topográfico das 21 seções é feito o cálculo hidráulico para estimativa do nível máximo da onda de ruptura em cada uma das seções transversais. A capacidade de transporte de cada seção transversal é determinada utilizando a fórmula de Manning-Strickler, definida pela equação 6 (Figura 1). 
Research, Society and Development, v. 10, n. 9, e11210917606, 2021

(CC BY 4.0) | ISSN 2525-3409 | DOI: http://dx.doi.org/10.33448/rsd-v10i9.17606

Figura 1 - Formulações definidas para o sistema aplicado.

\begin{tabular}{|c|c|}
\hline $\begin{array}{l}\text { Definicao da } \\
\text { Distáncia } \\
\left.\text { Maxima ( } D_{\text {mal }}\right) \\
\text { (eq. } 1)\end{array}$ & $D_{\max }=\left(8,87010^{-8} \times V_{\max }^{3}\right)-\left(2,60210^{-4} \times V_{\max }^{2}\right)+\left(2,64810^{-1} \times V_{\max }\right)+6,737$ \\
\hline $\begin{array}{l}\text { Vazäo Maxima } \\
\left(\mathrm{Q}_{\text {meg }}\right) \text { segundo } \\
\text { Froehlich } \\
\text { (eq. } 2)\end{array}$ & $Q_{\max }=0,607\left(V_{\max } 0.295 \times H_{\max }^{1,24}\right)$ \\
\hline 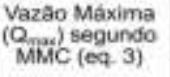 & $Q_{\max }=0,0039\left(V_{\max } 0,8122\right)$ \\
\hline $\begin{array}{l}\text { Vazão Máxima } \\
\text { à distáncia } x\end{array}$ & 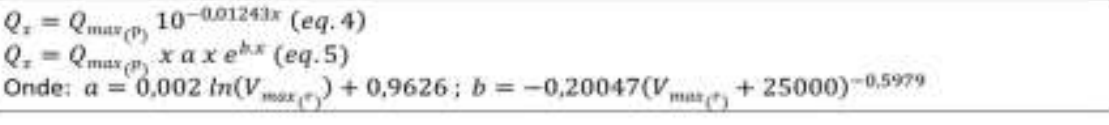 \\
\hline $\begin{array}{c}\text { Capacidade de } \\
\text { transporte } \\
\text { (eq, 6) }\end{array}$ & $Q \times / \sqrt{J}=K s \times A \times R^{2 / 3}$ \\
\hline $\begin{array}{l}\text { Onde: } V_{\max }-v \\
\text { momento da n } \\
a \text { e } b \text { - parame } \\
\text { do reservaróri } \\
\text { Manning-Stric } \\
\text { seçào de escoa }\end{array}$ & 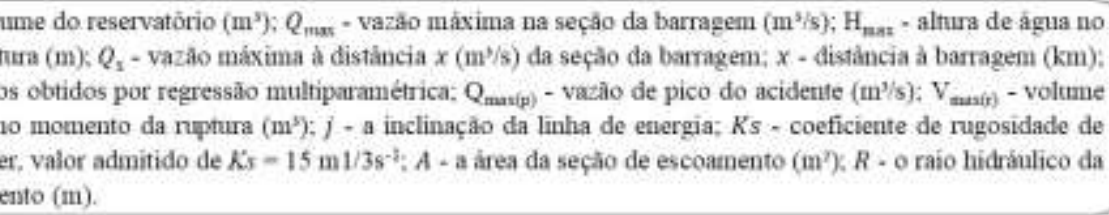 \\
\hline
\end{tabular}

Fonte: Autores.

Como resultado do cálculo hidráulico tem-se os níveis de água máximos atingidos em cada seção transversal e a partir deles pode-se criar um modelo digital por triangulação, e por diferença, pode ser delineada as áreas nas quais a altura da onda de ruptura é maior que a superfície da terra representada por um Modelo Digital de Elevação (MDE) gerado a partir de imagens do SRTM com resolução de $30 \mathrm{~m}$, resultando, portanto, nas áreas inundáveis. O processamento resumido do método LNEC é apresentado na Figura 2.

Figura 2 - Fluxograma de processamento do método do LNEC.
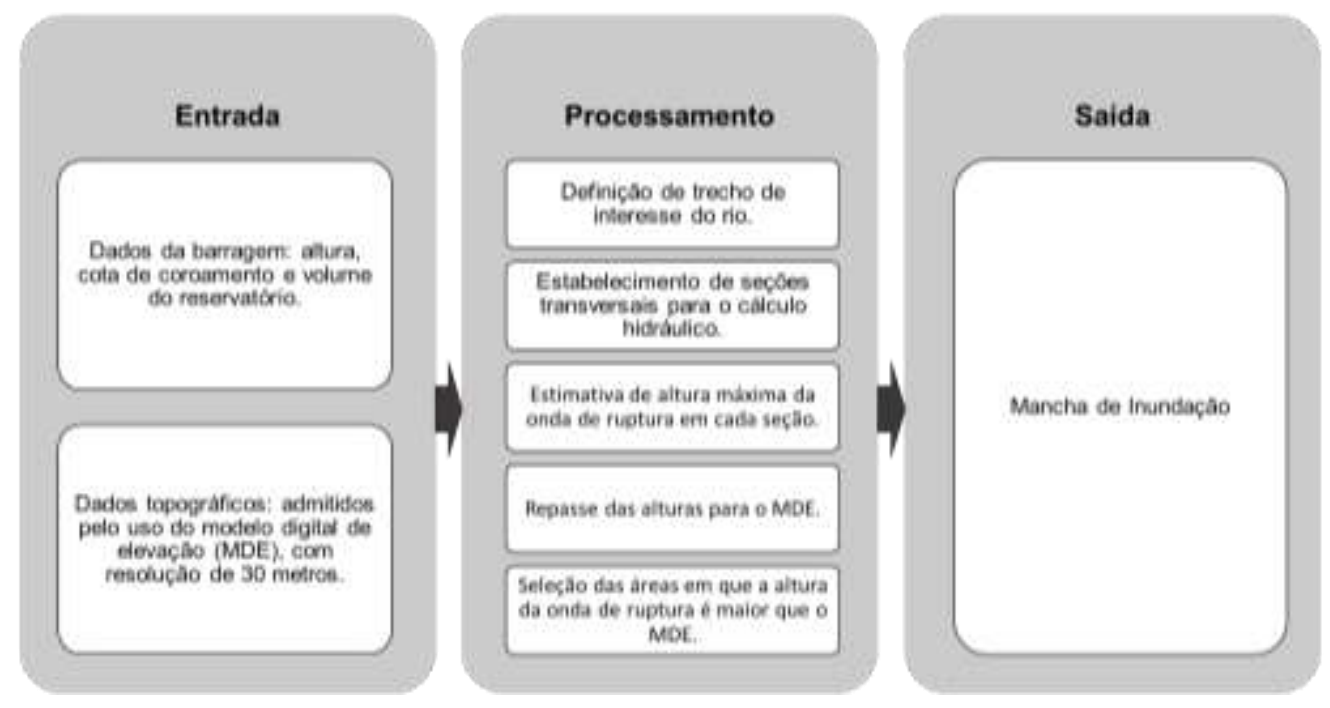

Fonte: Autores.

Cabe ressaltar que o SRTM já se encontra incorporado ao processamento automático do método do LNEC, ou seja, 
não é necessário baixar, nem processar nenhuma imagem, pois esse MDE já vem junto com os demais arquivos disponibilizados para executar o modelo. Basta informar a zona UTM (fuso e hemisfério) na qual a barragem está localizada. Com relação a cota de coroamento, esse dado é obtido pelo somatório da altura da estrutura de barramento, com a cota do SRTM do ponto de início da modelagem.

As questões associadas aos MDE's gerados a partir de imagens SRTM compreenderem principalmente dois componentes, o horizontal, frequentemente referido como a precisão dos componentes $\mathrm{X}$ e $\mathrm{Y}$, e o componente vertical ou precisão do atributo; na avaliação do modelo LNEC foram consideradas as influências da vegetação e das formas de cobertura da terra, Elkhrachy (2018) recomenda que os dados de elevação SRTM de $30 \mathrm{~m}$ sejam usados na elaboração de cartografias com intervalo de contorno não inferior a $16 \mathrm{~m}$.

Destaca-se também, que apenas o volume de líquido descarregado e os dados altimétricos do terreno são considerados para indicar a extensão da mancha gerada. Outros fatores que poderiam interferir na onda de propagação não são considerados por essa metodologia, tais como: viscosidade do material retido na barragem, velocidade do escoamento, índice pluviométrico da região, formas de uso da terra, características estruturais da barragem e propriedades morfométricas da bacia de drenagem. Este destaque é importante, pois reforça o objeto da aplicação do método ser indicativa para o processo de tomada de decisão, principalmente no subsídio à implantação de sistemas de monitoramento e alerta.

\section{Metodologia}

A área de estudo se encontra na região oeste do município de Barcarena (PA), nas proximidades do Igarapé Murucupi, afluente do Furo do Arrozal, que por sua vez desemboca na Baía de Marajó. A jusante do depósito de resíduos sólidos (DRS1) são localizadas as comunidades de Itupanema, Vila dos Cabanos e Laranjal (Figura 3).

Figura 3 - Mapa de localização da área de estudo.

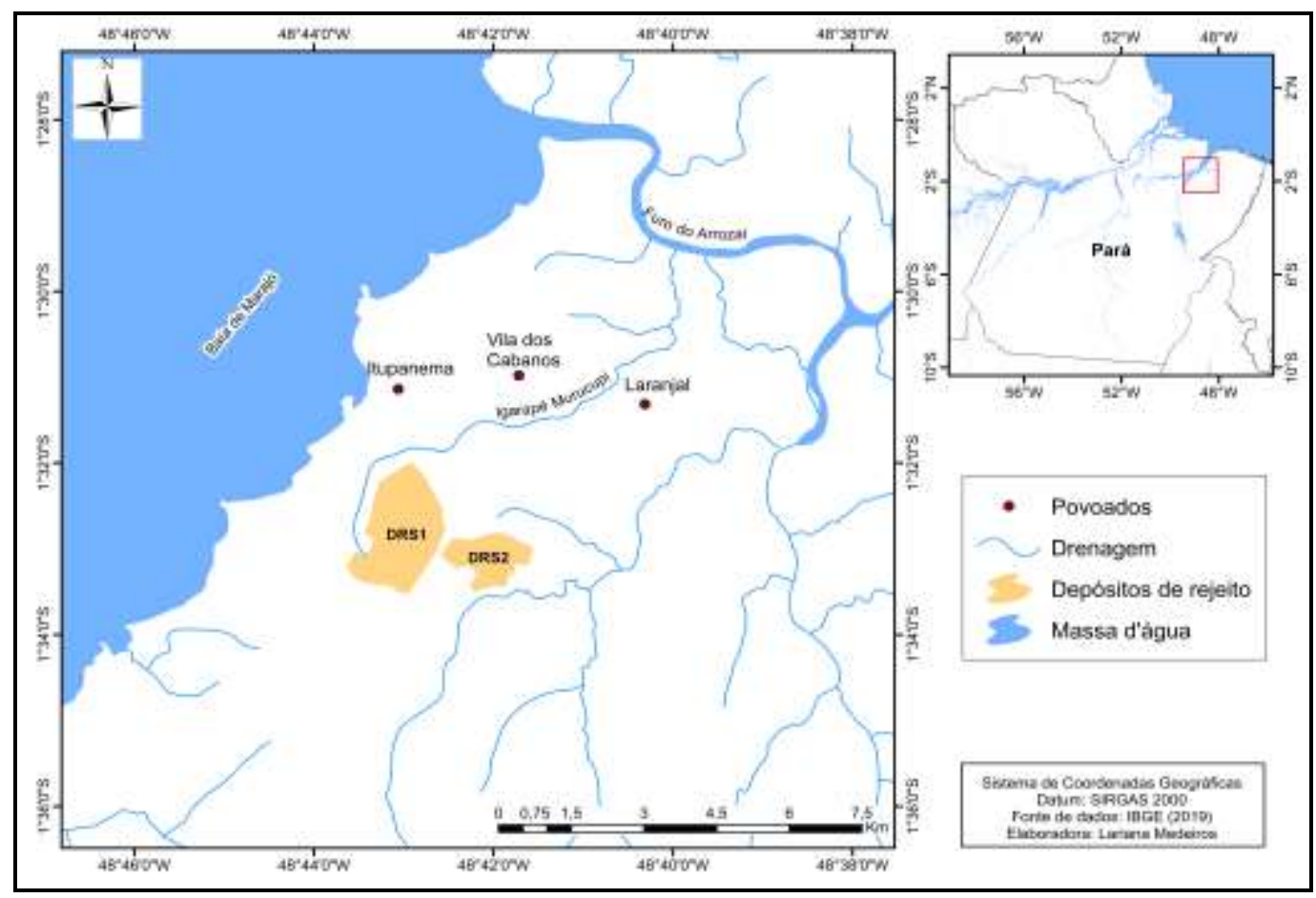

Fonte: Autores.

O DRS1 abriga resíduos resultantes da produção de alumina na região. Tais resíduos são comumente chamados de 
lama vermelha. O DRS1 está em operação há 24 anos, possui capacidade de armazenamento de $51,70 \mathrm{Mm}^{3}$ e volume armazenado de 47,90 $\mathrm{Mm}^{3}$. Antes de ser encaminhado para o depósito, o rejeito passa por 8 filtros prensa, gerando um resíduo seco com 78\% de teor de sólidos, que é empilhado e compactado (Alunorte, 2021). Rivas Mercury et al. (2010) informa que a lama vermelha é um resíduo semissólido alcalino composto essencialmente por sólidos insolúveis provenientes da bauxita, tais como, óxidos e hidróxidos de ferro (hematita e goethita), de titânio (anatásio) e de alumínio (gibbsita, boemita e diásporo), sílica (quartzo) e outras impurezas. Ainda segundo o autor, devido a elevada alcalinidade, com pH na faixa de 11,9 e 13,6, a lama vermelha pode ser classificada, de acordo com a NBR 10.004/2004, como resíduo Classe I (perigoso por apresentar as propriedades de corrosividade e reatividade).

A pesquisa realizada foi de natureza quantitativa com a definição de elementos métricos de caracterização da possível área de influência (Koche, 2011). Considerando o procedimento de análise, o estudo foi desenvolvido em 3 etapas: Préprocessamento, Simulação e Análise de Risco, as quais foram realizadas em software específico de geoprocessamento. Na etapa de Pré-processamento foram realizados os procedimentos necessários para preparação das análises posteriores, tais como, levantamento bibliográfico sobre barragens de rejeito de mineração, obtenção e organização de base vetorial (Tabela 1) e matricial (Tabela 2).

Tabela 1 - Características da base vetorial utilizada.

\begin{tabular}{c|c|c|c}
\hline Tipo & Produto & Escala & Fonte \\
\hline Rede de drenagem & $\begin{array}{c}\text { Identificação dos corpos hídricos da } \\
\text { área }\end{array}$ & $1: 250.000$ & $\begin{array}{c}\text { ANA (2019) e IBGE } \\
(2019)\end{array}$ \\
\hline Localidades & Caracterização e análise da área & $1: 250.000$ & IBGE (2019) \\
\hline Rodovias & $\begin{array}{c}\text { Análise de impactos } \\
\text { socioeconômicos }\end{array}$ & $1: 250.000$ & IBGE (2019) \\
\hline
\end{tabular}

Fonte: Autores.

Tabela 2 - Características da base matricial utilizada.

\begin{tabular}{c|c|c|c}
\hline Tipo & Produto & $\begin{array}{c}\text { Resolução } \\
\text { espacial }\end{array}$ & Fonte \\
\hline $\begin{array}{c}\text { Imagens SRTM (Shuttle Radar } \\
\text { Topography Mission) }\end{array}$ & $\begin{array}{c}\text { Geração do Modelo Digital de } \\
\text { Elevação }\end{array}$ & $30 \mathrm{~m}$ & $\begin{array}{c}\text { Farr e Kobrick (2000) e } \\
\text { Farr et al. (2007) }\end{array}$ \\
\hline $\begin{array}{c}\text { Imagens de Satélite do Google Earth } \\
\text { Pro }\end{array}$ & Caracterização da área & $2,8 \mathrm{~m}$ & Maxar (2020) \\
\hline
\end{tabular}

Fonte: Autores.

Posteriormente, foi realizada a Simulação da inundação em caso de vazamento hipotético de rejeitos do DRS1, utilizando o método do LNEC, que precisou atender as seguintes adequações, dada sua aplicação associada a barramentos:

(1) Com relação ao ponto de início para a modelagem, que seria a barragem, foi necessário escolher o ponto que se julgou mais adequado entre os quatro diques que delimitam o depósito de rejeitos.

(2) Ao invés de se utilizar o volume total do reservatório para um caso de ruptura, optou-se por avaliar condições de extravasamento de acordo com diferentes volumes de descarregamento, uma vez que esta é uma situação mais recorrente no caso de depósitos de rejeitos. Os testes visaram a avaliação da adequação da mancha ao MDE da região, tendo a lógica da conformação topográfica, partindo do princípio que este seria o único fator interveniente.

A partir dos testes realizados verificou-se que foi possível gerar manchas considerando uma altura da estrutura de barramento de $20 \mathrm{~m}$ e os volumes de descarga de rejeito no intervalo entre $1 \mathrm{hm}^{3}$ a $480 \mathrm{hm}^{3}$. Os resultados indicaram que o valor igual a $1 \mathrm{hm}^{3}$ subdimensionou a mancha e igual ou superior a $480 \mathrm{hm}^{3}$ apresentou incoerências ou erros de processamentos. Desta forma, os testes tiveram uma melhor adequação com volumes estimados de rejeito de $10 \mathrm{hm}^{3}, 100 \mathrm{hm}^{3}$ 
e $400 \mathrm{hm}^{3}$.

A fim de gerar manchas que representassem diferentes graus de risco (alto, médio e baixo) foram geradas três simulações (volumes de $10 \mathrm{hm}^{3}, 100 \mathrm{hm}^{3}$ e $400 \mathrm{hm}^{3}$ ) com critérios semelhantes: altura de $20 \mathrm{~m}$, cota de coroamento de $41 \mathrm{~m}$, mesmo corpo hídrico de referência e seções similares. A Tabela 3 apresenta resumidamente os dados de entrada que foram utilizados nas análises.

Tabela 3 - Dados de entrada utilizado nas análises.

\begin{tabular}{l|c|c|c}
\hline \multicolumn{1}{c|}{ Dados de entrada } & B010 & B100 & B400 \\
\hline Altura da estrutura de barramento $(\mathrm{m})$ & 20 & 20 & 20 \\
\hline Cota de coroamento $(\mathrm{m})$ & 41 & 41 & 41 \\
\hline Volume liberado $\left(\mathrm{hm}^{3}\right)$ & 10 & 100 & 400 \\
\hline
\end{tabular}

Fonte: Autores.

Após a geração automática, as manchas passaram por uma etapa de edição para retirada de polígonos isolados, preenchimento de espaços vazios dentro da mancha gerada e ajuste de áreas molhadas, fazendo um buffer de $50 \mathrm{~m}$ entorno do Igarapé Murucupi. Como ponto inicial da modelagem, considerou-se o local em que o Igarapé Murucupi intercepta um dos taludes do DRS1 (Figura 4). Como a extensão do corpo hídrico a ser analisada, foi utilizado o comprimento do Igarapé Murucupi. Por fim, foi feita a Análise dos Riscos relacionados ao vazamento dos rejeitos de alumina na área de abrangência do Igarapé Murucupi.

Figura 4 - Ponto considerado para início da modelagem em destaque.

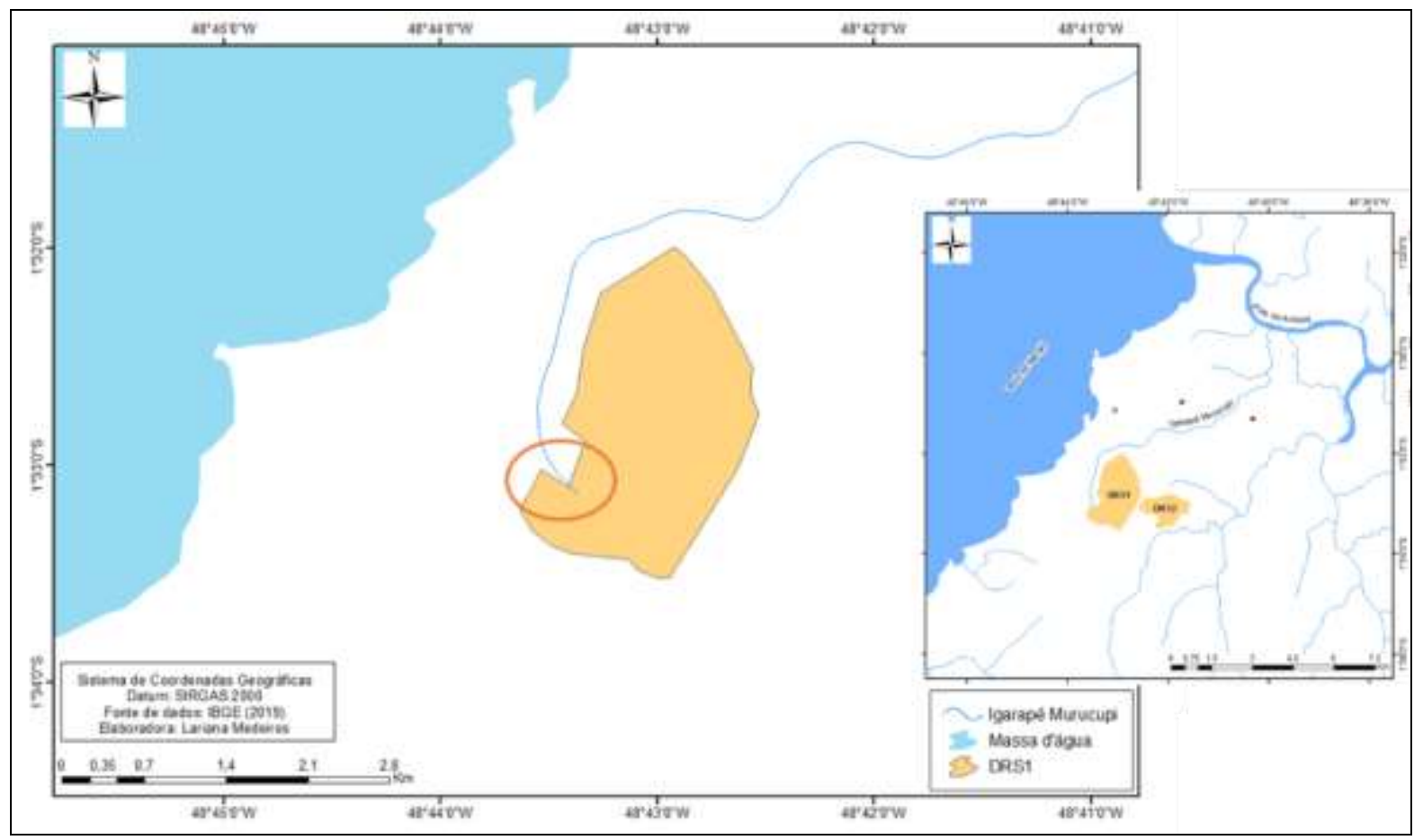

Fonte: Autores.

\section{Resultados e Discussão}

As simulações realizadas com os volumes de descarregamento de $10 \mathrm{hm}^{3}, 100 \mathrm{hm}^{3}$ e $400 \mathrm{hm}^{3}$ resultaram nas manchas 
de inundação mostradas na Figura 5. Em alguns pontos das manchas de $100 \mathrm{hm}^{3} \mathrm{e} 400 \mathrm{hm}^{3}$, o delineamento ficou retilíneo, o que se deve às seções transversais, utilizadas na metodologia adotada, para obter as cotas de interesse para o estudo, sendo a mancha obtida por meio da diferença entre altura máxima da onda de cheia e os valores do MDE. Portanto, o traçado da mancha acompanha os pontos da seção nos quais a altura máxima da onda de cheia foi superior ao valor do MDE.

Figura 5 - Manchas com as respectivas seções e com o corpo hídrico editado.

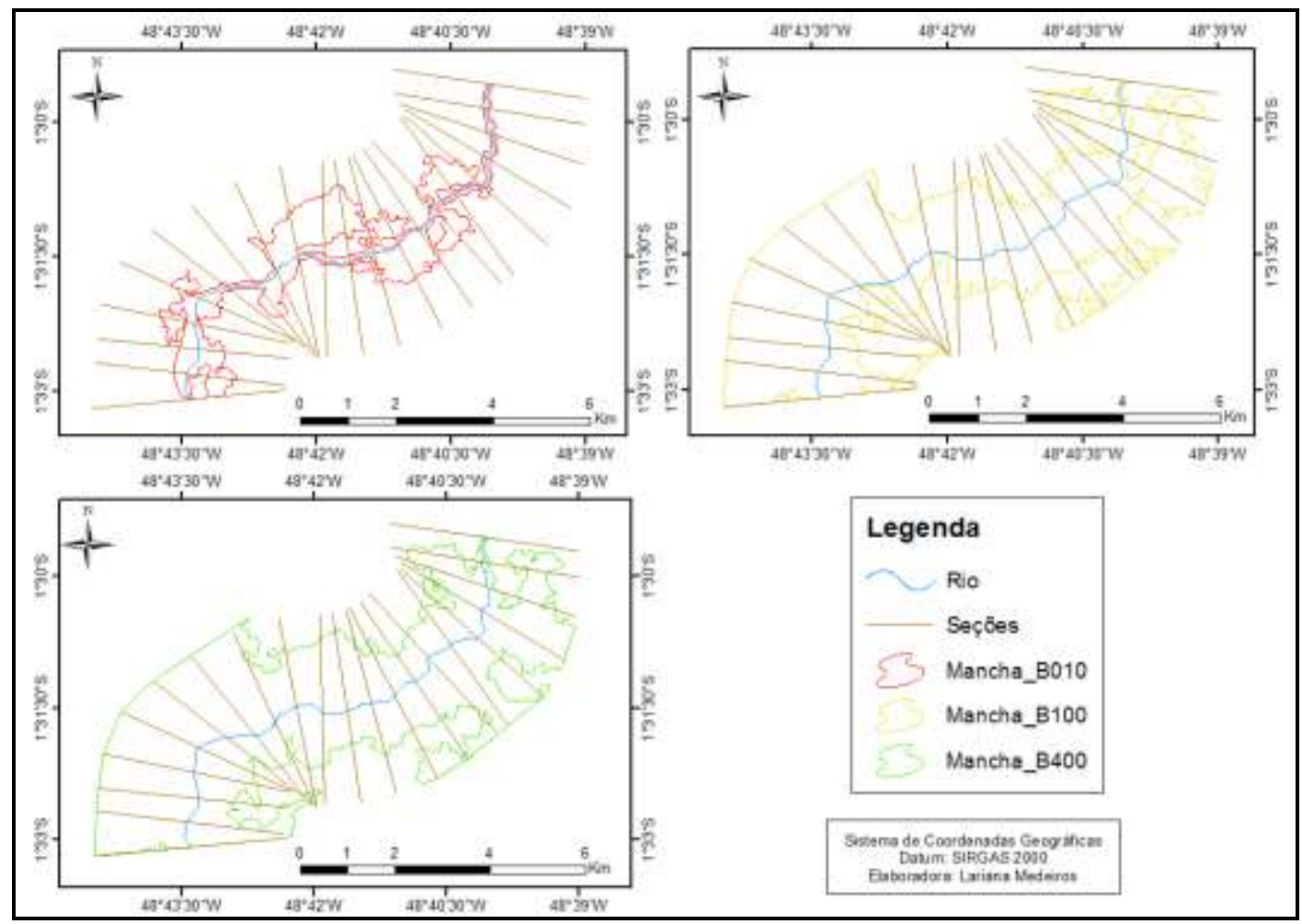

Fonte: Autores.

O MDE, obtido através da imagem SRTM, é a base para a geração da mancha e permite verificar as cotas que as manchas de inundação alcançaram: a B010 atingiu a cota 27m, a B100 até 29m e a B400 até 31m (Figura 6a). Observa-se que em certos pontos a mancha se delineia excluindo as áreas com cotas mais elevadas. Rodrigues et al. (2011) argumentam sobre a qualidade planialtimétrica dos dados SRTM, havendo a presença de deslocamento da componente vertical, que pode ser parcialmente atenuado durante o processamento dos modelos, porém, destacam o efeito da vegetação na altimetria gerada.

Desta forma, as manchas apresentadas na Figura 6, devem ser tratadas como áreas de influência norteadoras da análise, uma vez que outros componentes topográficos, de cobertura da terra e de tipologia do rejeito, devem ser considerados no seu detalhamento. Tschiedel e Paiva (2018) ao analisarem estudos de rompimentos de barragens alterando diferentes dados de entrada, observaram que em alguns casos foi possível compensar as incertezas relativas à topografia de um MDE mais impreciso a partir da adoção de uma superestimativa do volume do reservatório, em favor da segurança.

Com base nas manchas geradas foi produzido o mapa de risco a vazamentos hipotéticos do depósito de rejeitos DRS1, considerando alto, médio e baixo risco à inundação ao longo do Igarapé Murucupi (Figura 6b). Santos et al. (2020) destacam a importância destes modelos de simulação, principalmente no controle do processo de urbanização das áreas proximais aos empreendimentos minerários, cujo avanço pode acarretar no incremento da vulnerabilidade social, pela proximidade da área de influência estimada. 
Research, Society and Development, v. 10, n. 9, e11210917606, 2021

(CC BY 4.0) | ISSN 2525-3409 | DOI: http://dx.doi.org/10.33448/rsd-v10i9.17606

Figura 6 (a) e (b) - Cartografia derivada dos modelos gerados, com a área de inundação estimada.
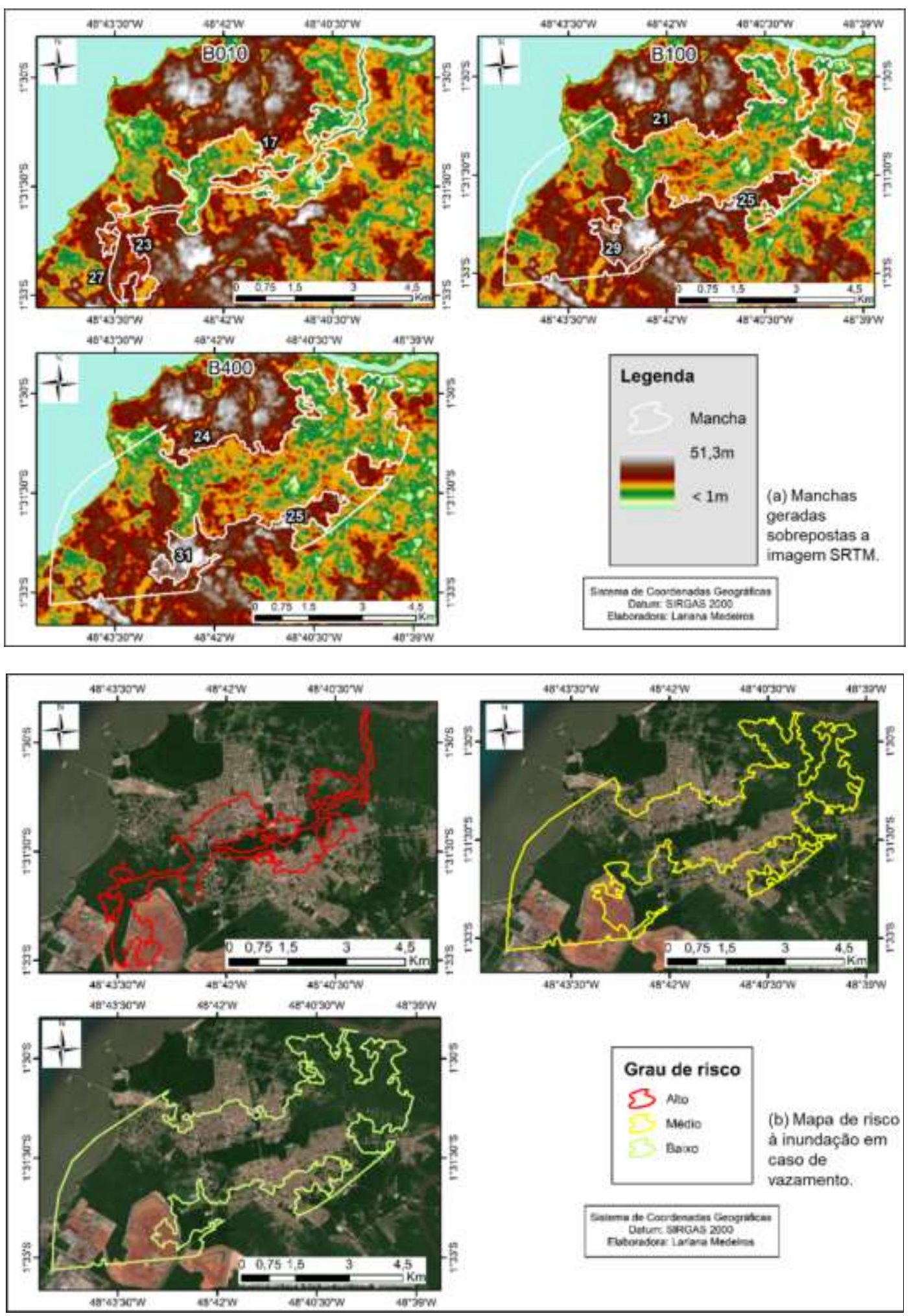

Fonte: Autores.

Pereira et al. (2017) utilizaram o modelo desenvolvido pelo LNEC para analisar 28 barragens com volumes de reservatório e altura variáveis e constataram que a principal vantagem desse método é a rapidez na obtenção dos resultados referentes à área inundada e ao caudal de pico. Sendo possível de ser aplicado, num curto período de tempo, a um grande número de barragens, permitindo a classificação das estruturas em termos de risco, utilizando para isso programas ou sistemas 
de acesso livre.

A fim de melhor evidenciar os possíveis danos ambientais e socioeconômicos decorrentes de um hipotético vazamento ou rompimento no DRS1, foi elaborada a Figura 7, onde é possível visualizar as comunidades e os trechos rodoviários localizados na área de abrangência da mancha. Destaca-se a presença das comunidades de Itupanema, Laranjal e Vila dos Cabanos, que representam áreas diretamente vinculadas ao desenvolvimento das atividades econômicas da região associadas a atividade minerária.

Figura 7 - Povoados e rodovias localizados na área de estudo.

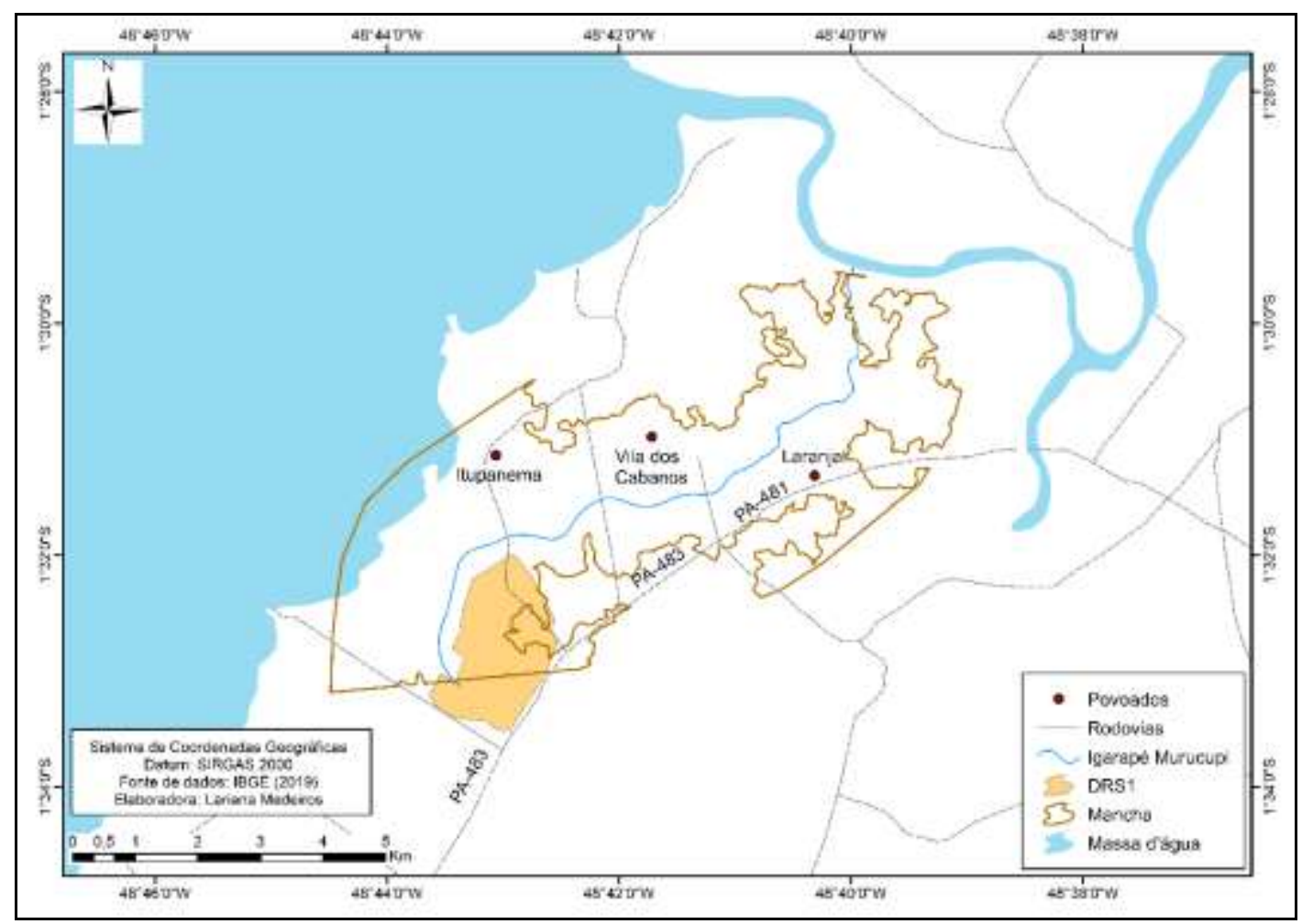

Fonte: Autores.

O impacto ambiental decorrente do descarregamento do rejeito tem o agravante da lama vermelha, que pode ser classificada como um resíduo perigoso, por possuir elevada alcalinidade, e consequentemente poder apresentar propriedades de corrosividade e reatividade. Rocha (2015) evidencia que mesmo com as incertezas associadas aos estudos de rompimento de barragens, estes, juntamente com as análises de risco, são de extrema importância para o conhecimento da ordem de grandeza dos danos da inundação e devem sempre ser realizados, uma vez que o desconhecimento total dos danos potenciais gera mais insegurança ao empreendedor e à sociedade do que a noção de um custo de inundação, com suas respectivas incertezas.

Lemos e Pimentel (2021) realizaram o levantamento de desastres com vazamentos registrados nos inquéritos policiais de 2003 a 2018 na região que envolve a área de influência delimitada na Figura 7, e registraram 12 ocorrências, cujo resultado das análises e conclusão das investigações indicaram como danos mais recorrentes a poluição hídrica pelo vazamento de efluentes alcalinos (lama vermelha) e pelo lançamento de caulim no solo, na praia e nos cursos d'água.

Steinbrenner et al. (2020) descrevem o ocorrido em 17 de fevereiro de 2018, onde após um evento de precipitação pluviométrica, acima da média para o período (173 mm em apenas 12 horas), ocorreu o deslocamento de material tóxico no rio Pará e seus afluentes, atingindo as comunidades locais. Santos et al. (2017) reforçam a atenção para a região, que segundo os autores apresenta um Índice de Vulnerabilidade Climática (IVC) igual a 0,57, ficando atrás de Belém (1) e Ananindeua $(0,71)$, 
este indicador deriva da porcentagem de meses com precipitação extrema alta, o que traduz um sinal de alerta para a região, principalmente para um monitoramento mais efetivo associado ao período chuvoso. E mesmo durante o menos chuvoso, este deve se manifestar, pois conforme o registrado por Lemos e Pimentel (2021), as ocorrências não ficaram limitadas ao período de maior intensidade de chuvas.

Tais evidências, reforçam a necessidade de se considerar o mapeamento apresentado na Figura 6 como norteador para o planejamento territorial no município de Barcarena, pois como destacam Rodrigues et al. (2020) a sede municipal e o Distrito de Murucupi, onde localizam-se a Vila Nova, a Vila São Francisco e a Vila dos Cabanos, além das ocupações de Itupanema, Pioneiro e Laranjal, e tantas outras pequenas comunidades, sofreram uma brusca alteração no perfil socioeconômico e demográfico a partir dos anos de 2000, principalmente com a ampliação da atuação de corporações transnacionais e de todo o setor terceirizado que surge para atender suas demandas. Assim, se a perspectiva é a ampliação da ocupação, potencializando a área do entorno do complexo mineral/industrial, faz-se necessária a implantação de ações preventivas à ocorrência de desastres e um intenso monitoramento integrado das condições ambientais, em destaque as climáticas, como subsídio a um sistema de alerta local.

Cabe destacar que a principal limitação percebida durante o desenvolvimento deste estudo foi a de não se ter um Modelo Digital de Elevação de melhor qualidade, sobretudo para que fosse possível comparar os resultados obtidos entre a mancha de inundação gerada utilizando o SRTM e um MDE mais refinado, levando-se em consideração ainda que se trata de uma região de baixa altitude como pode ser verificado por meio das cotas apresentadas na Figura 6a, o que pode dificultar ainda mais a utilização de um MDE de baixa qualidade.

\section{Conclusão}

A mineração é uma atividade de grande importância para a economia brasileira e o Pará está entre os principais polos minerais do país. Nesse contexto, Barcarena é um dos municípios paraenses que abriga grandes indústrias do setor mineral. Admitindo toda a ação de adequação às normas ambientais para armazenar os resíduos gerados pela atividade com segurança, o histórico de acidentes ambientais no município aponta que esta região é de alta vulnerabilidade à ocorrência de acidentes ambientais, principalmente em decorrência de eventos climáticos extremos.

Como forma de analisar esse risco, este estudo se propôs a gerar manchas de inundação considerando vazamentos hipotéticos, utilizando para isso a metodologia do LNEC. As manchas geradas adotando os volumes descarregados de $10 \mathrm{hm}^{3}$, $100 \mathrm{hm}^{3}$ e $400 \mathrm{hm}^{3}$ originaram, respectivamente, as zonas de alto, médio e baixo risco à inundação ao longo do Igarapé Murucupi, impactando diretamente as localidades de Vila dos Cabanos, Itupanema e Laranjal.

Os resultados obtidos, mesmo que dimensionados apenas considerando o indicador topográfico da região, são importantes, por mostrar que a metodologia simplificada, com ajustes, pode ser utilizada na geração da mancha de inundação para depósitos de rejeito e pela possibilidade de identificação de zonas de risco, indicando as áreas que devem ser priorizadas para ações do poder público, tendo como base ações preventivas e de monitoramento associado as estruturas de contenção de rejeitos.

Com relação a trabalhos futuros, sugere-se a realização de estudos utilizando a metodologia do LNEC adaptada para geração de manchas de inundação considerando outros depósitos de rejeito, a fim de identificar os possíveis danos relacionados a vazamentos ou rompimentos das estruturas e assim auxiliar na gestão de risco. Além disso, é importante a realização de pesquisas comparando o resultado da mancha gerada pela metodologia ajustada do LNEC com a mancha obtida usando um MDE de melhor qualidade, para que possam ser evidenciadas as diferenças práticas resultantes da aplicação de cada método. 


\section{Referências}

Alunorte - Alumina do Norte do Brasil S/A. (2021). Depósito de rejeitos. https://www.hydro.com/pt-BR/sobre-a-hydro/a-hydro-no-mundo/northamerica/brasil/barcarena/alunorte/deposito-de-residuos-solidos/

ANA - Agência Nacional de Águas. (2017). Geração de mancha para classificação de barragens quanto a dano potencial associado: metodologia simplificada. Brasília: Agência Nacional de Águas.

ANA - Agência Nacional de Águas. (2019). Metadados. https://metadados.ana.gov.br/geonetwork

ANM - Agência Nacional de Mineração. (2020). Anuário mineral brasileiro: principais substâncias metálicas. Brasília: Agência Nacional de Mineração.

Brasil. (2020). Lei n 14.066 , de 30 de setembro de 2020. Altera a Lei n 12.334 , de 20 de setembro de 2010, que estabelece a Política Nacional de Segurança de Barragens. Presidência da República. http://www.planalto.gov.br/ccivil_03/_ato2007-2010/2010/lei/112334.htm

Collischonn, W. \& Tucci, C. E. M. (1997). Análise do rompimento hipotético da Barragem de Ernestina. Revista Brasileira de Recursos Hídricos, 2 (2), 191206.

Elkhrachy, I. (2018) Vertical accuracy assessment for SRTM and ASTER Digital Elevation Models: A case study of Najran city, Saudi Arabia. Ain Shams Engineering Journal, 9 (4), 1807-1817. doi.org/10.1016/j.asej.2017.01.007

Farr, T. G. \& Kobrick, M. (2000). Shuttle radar topography mission produces a wealth of data. Amer. Geophys. Union Eos, 81 (48), $583-585$.

Farr, T. G., Rosen, P. A., Caro, E., Crippen, R., Duren, R., Hensley, S., Kobrick, M., Paller, M., Rodriguez, E., Roth, L., Seal, D., Shaffer, S., Shimada, J., Umland, J., Werner, M., Oskin, M., Burbank, D. \& Alsdorf, D. (2007). The shuttle radar topography mission. Rev. Geophys., 45, RG2004. doi.org/10.1029/2005RG000183

IBGE - Instituto Brasileiro de Geografia e Estatística. (2019). Bases cartográficas contínuas. ftp://geoftp.ibge.gov.br/.

Koche, J. C. (2011). Fundamentos de metodologia científica. Vozes.

Kumar, S., Jaswal, A., Pandey, A. \& Sharma, N. (2017). Literature review of Dam Break studies and inundation mapping using hydraulic models and GIS. International Research Journal of Engineering and Technology, 4 (5), 55-61.

Lemos, M. A. Q. \& Pimentel, M. A. S. (2021). Mineração e desastres ambientais com rejeitos de bauxita e caulim no município de Barcarena-Pará-BrasilAmazônia. Territorium, 28 (I), 137-156. doi.org/10.14195/1647-7723_28-1_8

Maxar - Maxar Technologies. (2020). www.maxar.com.

Pará. (2018). Relatório final da comissão externa das bacias de rejeitos de mineração em Barcarena/PA. Belém: Assembleia Legislativa do Estado do Pará.

Pereira, C. E., Viseu, M. T., Melo, J. F., Martins, T., Salla, M. R. \& Mota, K. R. R. (2017). Comparação entre modelos simplificados e o modelo HEC-RAS no estudo de áreas de inundação para o caso de Minas Gerais, Brasil. Revista Recursos Hídricos, 38 (1), 75-90. doi.org/10.5894/rh38n1-cti3

Ribeiro, J. C. J. \& Silva, L. C. N. (2018). A mineração no Estado do Pará e as barragens de rejeito: o paradigma entre a exploração e os impactos negativos decorrentes. Congresso Internacional de Direito Ambiental, Belo Horizonte, MG, Brasil, 5.

Rivas Mercury, J. M., Galdino, L. G., Vasconcelos, N. S. L. S., Paiva, A. E. M., Cabral, A. A. \& Angélica, R. S. (2010). Estudo do comportamento térmico e propriedades físico-mecânicas da lama vermelha. Revista Matéria, 15 (3), 445-460. doi.org/10.1590/S1517-70762010000300007

Rocha, F. F. (2015). Retroanálise da ruptura da barragem São Francisco - Miraí, Minas Gerais, Brasil (Dissertação de Mestrado). Universidade Federal de Minas Gerais, Belo Horizonte, MG, Brasil.

Rodrigues, T. G., Paradella, W. R. \& Oliveira, C. G. (2011). Evaluation of the altimetry from SRTM-3 and planimetry from high-resolution PALSAR FBD data for semi-detailed topographic mapping in the Amazon Region. Anais da Academia Brasileira de Ciências, 83 (3), 953-966. doi.org/10.1590/S000137652011000300014

Rodrigues, J. C., Machado, R. L. M., Penha, L. R. \& Oliveira Neto, A. (2020). Interfaces do rural e do urbano na cidade de Barcarena, Amazônia Paraense. Revista InterEspaço, 06, 01-22. doi.org/10.18764/2446-6549.e202016

Rossi, C. L. C. U., Marques, M. G., Teixeira, E. D., Melo, J. F., Ferla, R. \& Prá, M. D. (2021). Dam-Break analysis: proposal of a simplified approach. Brazilian Journal of Water Resources, 26 (02), 1-15. doi.org/10.1590/2318-0331.262120200066

Santos, M. R. S., Vitorino, M. I. \& Pimentel, M. A. S. (2017). Vulnerabilidade e mudanças climáticas: análise socioambiental em uma mesorregião da Amazônia. Revista Ambiente \& Água, 12 (5), 842-854. doi.org/10.4136/ambi-agua

Santos, C. M., Silva, J. G., Salles, P. V., Braga, F. C. S. \& Poggiali, F. S. J. (2020). Análise da mancha de inundação da Barragem Sul da Mina de Brucutu, São Gonçalo do Rio Abaixo, MG. Revista Engenharia de Interesse Social, 5 (6), 35-52. doi.org/10.35507/25256041/reis.v5i6.5191

Simineral - Sindicato das Indústrias Minerais do Estado do Pará. (2019). $8^{\circ}$ Anuário Mineral do Pará. Belém: Sindicato das Indústrias Minerais do Estado do Pará.

Sousa, L. A., Araújo, S. M. S. \& Barbosa, M. F. N. (2021). Dam disruption in international scientific literature: A bibliometric analysis. Research, Society and Development, 10 (5), e16610514780. doi.org/10.33448/rsd-v10i5.14780 
Research, Society and Development, v. 10, n. 9, e11210917606, 2021

(CC BY 4.0) | ISSN 2525-3409 | DOI: http://dx.doi.org/10.33448/rsd-v10i9.17606

Steinbrenner, R. A., Neto, G. G., Bragança, P. L. \& Castro, E. M. R. (2020). Desastre da mineração em Barcarena, Pará e cobertura midiática: diferenças de duração e direcionamentos de escuta. Revista Eletrônica de Comunicação, Informação e Inovação em Saúde, 14 (2), $307-28$. doi.org/10.29397/reciis.v14i2.2063

Stela, L. H. P., Duarte, J. C. \& Pereira, C. O. (2020). Métodos de disposição dos rejeitos de minério de ferro alternativos ao método de barragens: uma revisão. Revista Brasileira de Processos Químicos, 1 (1), 1-58.

Tschiedel, A. F. \& Paiva, R. C. D. (2018). Uncertainty assessment in hydrodynamic modeling of floods generated by dam break. Brazilian Journal of Water Resources, 23 (30), 1-17. doi.org/10.1590/2318-0331.231820170074

Tschiedel, A. F., Paiva, R. C. D. \& Fan, F. M. (2020). Use of large-scale hydrological models to predict dam break-related impacts. Brazilian Journal of Water Resources, 25 (35), 1-18. doi.org/10.1590/2318-0331.252020190128 